\title{
ALMOST LINEARITY OF $\epsilon$-BI-LIPSCHITZ MAPS BETWEEN REAL BANACH SPACES
}

\author{
KIL-WOUNG JUN AND DAL-WON PARK
}

(Communicated by Palle E. T. Jorgensen)

\begin{abstract}
Let $X$ and $Y$ be real Banach spaces. A map $f$ between $X$ and $Y$ is called an $\epsilon$-bi-Lipschitz map if $(1-\epsilon)\|x-y\| \leq\|f(x)-f(y)\| \leq(1+\epsilon)\|x-y\|$ for all $x, y \in X$. In this note we show that if $f$ is an $\epsilon$-bi-Lipschitz map with $f(0)=0$ from $X$ onto $Y$, then $f$ is almost linear. We also show that if $f: X \longrightarrow Y$ is a surjective $\epsilon$-bi-Lipschitz map with $f(0)=0$, then there exists a linear isomorphism $I: X \rightarrow Y$ such that

$$
\|I(x)-f(x)\| \leq E(\epsilon, \alpha)\left(\|x\|^{\alpha}+\|x\|^{2-\alpha}\right)
$$

where $E(\epsilon, \alpha) \rightarrow 0$ as $\epsilon \rightarrow 0$ and $0<\alpha<1$.
\end{abstract}

\section{INTRODUCTION}

It is a well-known classical result of Mazur and Ulam [4] that an isometry $f$ from a real Banach space $X$ onto a real Banach space $Y$ with $f(0)=0$ is automatically linear. A map $f$ between Banach spaces $X$ and $Y$ is called an $(m, M)$-rigid map if

$$
m\|x-y\| \leq\|f(x)-f(y)\| \leq M\|x-y\|
$$

for all $x, y \in X$. We denote a $(1-\epsilon, 1+\epsilon)$-rigid map by $\epsilon$-bi-Lipschitz map. The following theorem follows from [1, Proposition 2].

Theorem. Let $f$ be an $\epsilon$-bi-Lipschitz map from a real Banach space $X$ onto a real Banach space $Y$, with $f(0)=0$ and $0 \leq \epsilon<\frac{1}{3}$. Then

$$
\|f(x+y)-f(x)-f(y)\| \leq c_{1}(3 \epsilon)^{c_{2}}(\|x\|+\|y\|), \text { for } x, y \in X
$$

where

$$
c_{1}=(8 / 3)\left((\alpha / 8)\left(\alpha^{2}+4 \alpha-1\right) /(\alpha-1)+1\right)
$$

and

$$
c_{2}=(\log (2 / \alpha))(\log ((\alpha+7) /(\alpha-1))+\log (2 / \alpha))^{-1}
$$

for $1<\alpha<2$.

Received by the editors August 8, 1994.

1991 Mathematics Subject Classification. Primary 46B20.

Key words and phrases. $\epsilon$-bi-Lipschitz map, almost linear map, real Banach spaces.

This work was partially supported by KOSEF, Grant No 91-08-00-01. 
Note that $c_{2}$ attains its maximum for $\alpha \in(1,2)$ at $\alpha=1.1572 \ldots$ and for this value of $\alpha, c_{1}$ and $c_{2}$ have values $5.5704 \ldots$ and $0.1216 \ldots$, respectively.

Jarosz [2] conjectured that if $f$ is an $\epsilon$-bi-Lipschitz map from a real Banach space $X$ onto a real Banach space $Y$, then $X$ and $Y$ are linearly isomorphic for sufficiently small $\epsilon$. Throughout, $R$ represents the real number field. There is an $\epsilon$-bi-Lipschitz map $f$ on $R$ which is not a linear map on $R$.

Example. We define an $\epsilon$-bi-Lipschitz map $f: R \rightarrow R$ such that

$$
f(x)= \begin{cases}(1+\epsilon) x, & \text { if } x \leq 2, \\ (1-\epsilon)(x-2)+2(1+\epsilon), & \text { if } x>2 .\end{cases}
$$

Throughout this paper, $X$ and $Y$ shall denote real Banach spaces. The $r$ neighborhood of a set $A$ is denoted by $B(A, r)$ and we abbreviate $B(\{x\}, r)$ and $B(\{0\}, r)$ by $B(x, r)$ and $B(r)$, respectively. The corresponding closed balls are denoted by $\bar{B}(x, r)$ and $\bar{B}(r)$. As usual, $[x, y]$ denotes the closed segment determined by $x$ and $y$. A bound subset $A$ of $X$ is said to be symmetric with respect to a point $a$ if $x$ in $A$ implies that $2 a-x$ is also in $A$. If $A$ is bounded and symmetric with respect to $a$, we define $\operatorname{rad} A$ to be $\inf \{r>0: A \subset B(a, r)\}$. We denote the Blaschke distance function on sets by $D$; that is, for $A_{1}, A_{2} \subset X$, $D\left(A_{1}, A_{2}\right)=\inf \left\{r>0: A_{1} \subset B\left(A_{2}, r\right)\right.$ and $\left.A_{2} \subset B\left(A_{1}, r\right)\right\}$. For $A \subset X$ and $\alpha>0$ we define $T(A, \alpha)=\{x \in X: A \subset \bar{B}(x, \alpha)\}=\bigcap\{\bar{B}(y, \alpha): y \in A\}$. For $x, y \in X$ we define $S(x, y, \alpha)=T([x, y], \alpha)=T(\{x, y\}, \alpha)=\bar{B}(x, \alpha) \cap \bar{B}(y, \alpha)$. We also define $C(A, \alpha)=A \cap T(A, \alpha)$. The following lemmas are due to Gevirtz [1] and John [3].

Lemma 1 [1, Lemma 2]. Let $x, y \in X$ and let $\beta, \gamma>|x-y| / 2$. Then

$$
D(S(x, y, \beta), S(x, y, \gamma)) \leq \frac{|\beta-\gamma|}{1-|x-y| / 2 \beta} .
$$

Lemma 2 [1, Lemma 3]. Let $\delta>0$. For every bounded convex symmetric subset $A \neq \varnothing$ of $X$ with center $a$ and all $\beta, \gamma$ for which $B(a, \delta) \subset C(A, \beta)$ and $\gamma>\operatorname{rad} A$ there holds

$$
D(C(A, \beta), C(A, \gamma)) \leq\left(1+\frac{\operatorname{rad} A}{\delta}\right)|\beta-\gamma| .
$$

Lemma 3 [1, Lemma 4]. Let $\delta>0$. For every bounded convex symmetric subset $A \neq \varnothing$ of $X$ with center $a$ and $F \subset X$ and $\beta$ for which $C(F, \beta) \neq \varnothing$ and $B(a, \delta) \subset$ $C(A, \beta)$ there holds

$$
D(C(A, \beta), C(F, \beta)) \leq\left(1+\frac{4 \operatorname{rad} A}{\delta}\right) D(A, F) .
$$

Lemma 4 [1, Lemma 5]. Let $A \subset U \subset X$ and let $f: U \longrightarrow Y$ be $(m, M)$-rigid. Then for all $\alpha>0$

$$
f(U \cap T(A, \alpha)) \subset f(U) \cap T(f(A), M \alpha) \subset f\left(U \cap T\left(A, \frac{M}{m} \alpha\right)\right) .
$$

Lemma 5 [3, Theorem II]. Let $f: X \rightarrow Y$ be $(m, M)$-rigid. Then $f(B(a, r)) \supset$ $B(f(a), m r)$. 


\section{The RESUlts}

Definition 6. A map $f$ from a real Banach space $X$ into a real Banach space $Y$ is an almost linear map if it satisfies

$$
\|f(x+y)-f(x)-f(y)\| \leq D(\epsilon)(\|x\|+\|y\|), \text { for } x, y \in X
$$

and

$$
\|f(\lambda x)-\lambda f(x)\| \leq D(\epsilon) E(\lambda)\|x\|, \text { for } \lambda \in R, x \in X
$$

where $D(\epsilon) \rightarrow 0$ as $\epsilon \rightarrow 0$ and $E(\lambda) \rightarrow 0$ as $\lambda \rightarrow 0$.

Let $x \neq y$ be points in $X$ and let $d=\|x-y\|$. For $\alpha>1$ we define $S_{n}=$ $S_{n}(x, y, d, \alpha)$ recursively as follows : $S_{1}=S\left(x, y, \frac{\alpha d}{2}\right)$ and, for $n \geq 1, S_{n+1}=$ $C\left(S_{n}, \frac{\alpha d}{2^{n}}+\sum_{i=1}^{n} \frac{1}{2^{i}}(\alpha-1) d\right)$.

Lemma 7. Let $x \neq y$ be points in $X, a=\frac{x+y}{2}$ and $\alpha>1$. Then for $n \geq 1, S_{n}$ is convex and symmetric with respect to $a$ and

$$
B\left(a, \frac{\alpha-1}{2} d\right) \subset S_{n} \subset B\left(a, \frac{\alpha d}{2^{n}}+\sum_{i=2}^{n} \frac{1}{2^{i}}(\alpha-1) d\right) .
$$

Proof. It is easy to verify that the assertion is true for $n=1$. Assume inductively that it is true for a given $n \geq 1$. The inductive hypothesis implies that $S_{n+1}$ is convex and symmetric with respect to $a$. If $z \in B\left(a, \frac{\alpha-1}{2} d\right)$ and $u \in S_{n}$, then

$$
\begin{aligned}
\|z-u\| & \leq\|z-a\|+\|a-u\| \\
& <\frac{\alpha-1}{2} d+\frac{\alpha}{2^{n}} d+\sum_{i=2}^{n} \frac{1}{2^{i}}(\alpha-1) d \\
& =\frac{\alpha}{2^{n}} d+\sum_{i=1}^{n} \frac{1}{2^{i}}(\alpha-1) d .
\end{aligned}
$$

This implies that $z \in S_{n+1}$. If $u \in S_{n+1}$, then $\|u-z\| \leq \frac{\alpha d}{2^{n}}+\sum_{i=1}^{n} \frac{1}{2^{i}}(\alpha-1) d$ for all $z \in S_{n}$. Since $S_{n+1}$ is symmetric with respect to $a, 2 a-u \in S_{n+1} \subset S_{n}$. Putting $2 a-u$ instead of $z$ in the above formula, we have $\|u-a\| \leq \frac{\alpha d}{2^{n+1}}+\sum_{i=2}^{n+1} \frac{1}{2^{i}}(\alpha-1) d$. That is, $\operatorname{rad} S_{n+1} \leq \frac{\alpha d}{2^{n+1}}+\sum_{i=2}^{n+1} \frac{1}{2^{i}}(\alpha-1) d$.

Proposition 8. Let $x, y \in X,\|x-y\|=d, \alpha>1$ and $0<m \leq M$. Let $f: X \longrightarrow Y$ be an $(m, M)$-rigid map. Writing $S_{n}, S_{n}^{\prime}$ and $\mu$ for $S_{n}(x, y, d, \alpha)$, $S_{n}(f(x), f(y), M d, \alpha)$ and $\frac{M}{m}$, respectively, there holds

$$
D\left(f\left(S_{n}\right), S_{n}^{\prime}\right) \leq K(m, M, \alpha)\|x-y\|
$$

where $K(m, M, \alpha)=M(\mu-1)\left(\frac{7 \alpha-1}{\alpha-1}\right)^{n-1}\left(\frac{31 \alpha^{3}-18 \alpha^{2}+10 \alpha-2}{(\alpha-1)(6 \alpha-4)}\right)$.

Proof. Replacing $U, A$ by $X,\{x, y\}$, respectively, in Lemma 4, we obtain

$$
f\left(S_{1}\right) \subset S_{1}^{\prime} \subset f(S(x, y, \alpha \mu d / 2)) .
$$


Since $S_{n+1}=C\left(S_{n}, \frac{\alpha d}{2^{n}}+\sum_{i=1}^{n} \frac{1}{2^{i}}(\alpha-1) d\right)$, Lemma 4 with $A=U=S_{n}$ implies that

$$
\begin{aligned}
f\left(S_{n+1}\right) & \subset C\left(f\left(S_{n}\right), M d\left[\frac{\alpha}{2^{n}}+\sum_{i=1}^{n} \frac{1}{2^{i}}(\alpha-1)\right]\right) \\
& \subset f\left(C\left(S_{n}, \mu d\left[\frac{\alpha}{2^{n}}+\sum_{i=1}^{n} \frac{1}{2^{i}}(\alpha-1)\right]\right)\right) .
\end{aligned}
$$

Formula (1) gives

$$
\begin{aligned}
D\left(f\left(S_{1}\right), S_{1}^{\prime}\right) & \leq D\left(f\left(S\left(x, y, \frac{\alpha d}{2}\right)\right), f\left(S\left(x, y, \frac{\alpha \mu d}{2}\right)\right)\right) \\
& \leq M D\left(S\left(x, y, \frac{\alpha d}{2}\right), S\left(x, y, \frac{\alpha \mu d}{2}\right)\right) .
\end{aligned}
$$

Lemma 1 with $\beta=\frac{\alpha d}{2}$ and $\gamma=\frac{\alpha \mu d}{2}$ implies that we have $D\left(f\left(S_{1}\right), S_{1}^{\prime}\right) \leq$ $M \alpha^{2} d \frac{(\mu-1)}{2(\alpha-1)}$. Put $D_{n}=D\left(f\left(S_{n}\right), S_{n}^{\prime}\right)$. Let $n \geq 1$ and

$$
W=C\left(f\left(S_{n}\right), M d\left[\frac{\alpha}{2^{n}}+\sum_{i=1}^{n} \frac{1}{2^{i}}(\alpha-1)\right]\right)
$$

Then (2) implies $W \neq \varnothing$, and so

$$
D_{n+1} \leq D\left(f\left(S_{n+1}\right), W\right)+D\left(S_{n+1}^{\prime}, W\right) .
$$

Formula (2) gives

$$
\begin{gathered}
D\left(f\left(S_{n+1}\right), W\right) \leq D\left(f\left(S_{n+1}\right), f\left(C\left(S_{n}, \mu d\left[\frac{\alpha}{2^{n}}+\sum_{i=1}^{n} \frac{1}{2^{i}}(\alpha-1)\right]\right)\right)\right) \\
=D\left(f\left(C\left(S_{n}, d\left[\frac{\alpha}{2^{n}}+\sum_{i=1}^{n} \frac{1}{2^{i}}(\alpha-1)\right]\right)\right),\right. \\
\left.f\left(C\left(S_{n}, \mu d\left[\frac{\alpha}{2^{n}}+\sum_{i=1}^{n} \frac{1}{2^{i}}(\alpha-1)\right]\right)\right)\right) .
\end{gathered}
$$

By Lemma 2 and Lemma 7,

$$
\begin{aligned}
D\left(f\left(S_{n+1}\right), W\right) \leq & d(\mu-1) M\left(1+\frac{\alpha d / 2^{n}+\sum_{i=2}^{n}(\alpha-1) d / 2^{i}}{(\alpha-1) d / 2}\right) \\
& \times\left[\frac{\alpha}{2^{n}}+\sum_{i=1}^{n} \frac{1}{2^{i}}(\alpha-1)\right] \\
\leq & d(\mu-1) M\left(1+\frac{\alpha}{\alpha-1}\right)\left(\frac{\alpha}{2}+\frac{\alpha-1}{2}\right) \\
\leq & d(\mu-1) M \frac{(2 \alpha-1)^{2}}{2(\alpha-1)} .
\end{aligned}
$$


Similarly, we may apply Lemma 3 with $a=\frac{f(x)+f(y)}{2}, A=S_{n}^{\prime}, F=f\left(S_{n}\right)$, $\beta=M\left[\frac{\alpha d}{2^{n}}+\sum_{i=1}^{n} \frac{1}{2^{i}}(\alpha-1) d\right]$ and $\delta=M \frac{\alpha-1}{2} d$.

$$
\begin{aligned}
D\left(S_{n+1}^{\prime}, W\right)= & D\left(C\left(S_{n}^{\prime}, M d\left[\frac{\alpha}{2^{n}}+\sum_{i=1}^{n} \frac{1}{2^{i}}(\alpha-1)\right]\right),\right. \\
& \left.C\left(f\left(S_{n}\right), M d\left[\frac{\alpha}{2^{n}}+\sum_{i=1}^{n} \frac{1}{2^{i}}(\alpha-1)\right]\right)\right) \\
\leq & \left(1+\frac{4\left(\alpha d / 2^{n}+\sum_{i=2}^{n}(\alpha-1) d / 2^{i}\right)}{(\alpha-1) d / 2}\right) D_{n} \\
\leq & \left(\frac{5 \alpha-1}{\alpha-1}\right) D_{n} .
\end{aligned}
$$

From (4) and (5) we get

$$
D_{n+1} \leq d(\mu-1) M \frac{(2 \alpha-1)^{2}}{2(\alpha-1)}+\left(\frac{5 \alpha-1}{\alpha-1}\right) D_{n} .
$$

Let $G=d(\mu-1) M \frac{(2 \alpha-1)^{2}}{2(\alpha-1)}, H=\frac{5 \alpha-1}{\alpha-1}$. By induction we then get

$$
\begin{aligned}
D_{n} & \leq G\left(1+H+\cdots+H^{n-2}\right)+H^{n-1} D_{1} \\
& =G\left(\frac{H^{n-1}-1}{H-1}\right)+H^{n-1} D_{1} \\
& \leq(\mu-1) M\left(\frac{5 \alpha-1}{\alpha-1}\right)^{n-1}\left(\frac{8 \alpha^{3}-8 \alpha^{2}+5 \alpha-1}{8 \alpha(\alpha-1)}\right)\|x-y\| .
\end{aligned}
$$

Proposition 9. Let $x, y \in X, 0<m \leq M, \mu=\frac{M}{m}<2$. Let $f: X \longrightarrow Y$ be $(m, M)$-rigid. Then

$$
\left\|\frac{f(x)+f(y)}{2}-f\left(\frac{x+y}{2}\right)\right\| \leq c_{1}(\mu-1)^{c_{2}} M\|x-y\|
$$

where $c_{1}=\frac{3 \alpha-1}{2}+\frac{(9 \alpha-5)\left(17 \alpha^{3}-25 \alpha^{2}+16 \alpha-4\right)}{8(2 \alpha-1)(\alpha-1)^{2}}$ and $c_{2}=\frac{\log 2}{\log \frac{2(9 \alpha-5)}{\alpha-1}}$ for $\alpha>1$.

Proof. Put $a=\frac{x+y}{2}$ and $p=\frac{f(x)+f(y)}{2}$. By Lemma 5 and Lemma 7, we obtain $B\left(f(a), m \frac{\alpha-1}{2} d\right) \subset f\left(S_{n}\right)$ and $S_{n}^{\prime} \subset B\left(p, M \frac{\alpha d}{2^{n}}+M \frac{\alpha-1}{2} d\right)$. Since $D_{n}=$ $D\left(f\left(S_{n}\right), S_{n}^{\prime}\right), f\left(S_{n}\right) \subset B\left(S_{n}^{\prime}, D_{n}\right)$. Thus we have

$$
\begin{aligned}
B\left(f(a), m \frac{\alpha-1}{2} d\right) & \subset B\left(B\left(p, M \frac{\alpha d}{2^{n}}+M \frac{\alpha-1}{2} d\right), D_{n}\right) \\
& =B\left(p, M \frac{\alpha d}{2^{n}}+M \frac{\alpha-1}{2} d+D_{n}\right) .
\end{aligned}
$$

Hence Proposition 8 implies

$$
\begin{aligned}
\|f(a)-p\| \leq & (M-m) \frac{\alpha-1}{2} d+M \frac{\alpha d}{2^{n}} \\
& +d(\mu-1) M\left(\frac{5 \alpha-1}{\alpha-1}\right)^{n-1} \frac{8 \alpha^{3}-8 \alpha^{2}+5 \alpha-1}{8 \alpha(\alpha-1)} .
\end{aligned}
$$


Let $E=\frac{1}{2}$ and $F=\frac{5 \alpha-1}{\alpha-1}$. For given $\alpha, \mu$ we use an integer $n$ which is chosen in such a way that the last expression takes the form $c_{1}(\mu-1)^{c_{2}} M\|x-y\|$, apart from negligible differences. Explicitly, we write $n$ in the form

$$
n=-\beta(\log (\mu-1)) / \log F+\xi+1
$$

where $0<\beta<1$ and $0 \leq \xi<1$. Since $\mu<2$, we have that $n \geq 1,(\mu-1) F^{n-1}=$ $(\mu-1)^{1-\beta} F^{\xi} \leq(\mu-1)^{1-\beta} F$ and $E^{n}=(\mu-1)^{-\beta \log \frac{1}{2} / \log F} E^{\xi} \leq(\mu-1)^{\beta \log 2 / \log F}$. If $\beta$ is determined so that $1-\beta=\beta \log 2 / \log F$, then $\beta=\frac{\log \bar{F}}{\log 2+\log F}$. Put $c_{2}=1-\beta$. Then

$$
\begin{aligned}
d M[\alpha & \left.\left(\frac{1}{2}\right)^{n}+(\mu-1)\left(\frac{5 \alpha-1}{\alpha-1}\right)^{n-1} \frac{8 \alpha^{3}-8 \alpha^{2}+5 \alpha-1}{8 \alpha(\alpha-1)}\right] \\
& \leq d M(\mu-1)^{c_{2}}\left(\alpha+\frac{(5 \alpha-1)\left(8 \alpha^{3}-8 \alpha^{2}+5 \alpha-1\right)}{8 \alpha(\alpha-1)^{2}}\right) .
\end{aligned}
$$

Since $0<c_{2}<1$ and $\mu \leq 2$

Thus

$$
(M-m) \frac{\alpha-1}{2} d \leq M(\mu-1)^{c_{2}} \frac{\alpha-1}{2} d .
$$

$$
\|f(a)-p\| \leq M(\mu-1)^{c_{2}}\left(\frac{3 \alpha-1}{2}+\frac{(5 \alpha-1)\left(8 \alpha^{3}-8 \alpha^{2}+5 \alpha-1\right)}{8 \alpha(\alpha-1)^{2}}\right)\|x-y\| .
$$

Remark. It is easy to show that $c_{2}$ converges to $\log 2$ as $\alpha$ converges to infinity. If we choose $\alpha=10$, then $c_{1}=6.9314 \ldots$ and $c_{2}=0.2902 \ldots$ We denote these values by $K_{1}$ and $K_{2}$, respectively. The later value is greater than $0.1216 \ldots$ which is the maximum value of $c_{2}$ in [1, Proposition 2]. If $\mu \geq 2$, there exists $\delta>0$ such that $\left\|\frac{1}{2}(f(x)+f(y))-f\left(\frac{x+y}{2}\right)\right\| \leq \delta\|x-y\|$, for $x, y \in X$.

Lemma 10. Let $f: X \longrightarrow Y$ be a continuous map such that $\| \frac{1}{2}(f(x)+f(y))-$ $f\left(\frac{x+y}{2}\right)\|\leq K\| x-y \|$, for some $K>0$ and for all $x, y \in X$, with $f(0)=0$. Then

$$
\|f(\lambda x)-\lambda f(x)\| \leq 2 K\|x\|, \quad 0 \leq \lambda \leq 1, x \in X .
$$

Proof. Let $Q_{n}=\left\{\frac{p}{2^{n}} \mid p=1,2, \ldots, 2^{n}\right\}$ and $Q=\bigcup_{n=1}^{\infty} Q_{n}$. Then $Q$ is dense in $\{\lambda \mid 0 \leq \lambda \leq 1\}$. Since $f$ is continuous, it is sufficient to show that $\|\lambda f(x)-f(\lambda x)\| \leq$ $\sum_{i=0}^{n-1} \frac{1}{2^{i}} K\|x\|$ for all $x \in X, \lambda \in Q_{n}$. We prove the above formula by induction. It is clearly true for $n=1$, and we assume it is true if we have $Q_{n-1}$, for $n \geq 2$. We take $\lambda \in Q_{n}, \lambda=\frac{p}{2^{n}}$. If $p$ is divided by 2 , then $\lambda \in Q_{n-1}$. Otherwise there exists an integer $r$ such that $p=2 r+1$ and so $r \leq 2^{n-1}-1$. Thus $\frac{r}{2^{n-1}}, \frac{r+1}{2^{n-1}} \in Q_{n-1}$. Hence we obtain

$$
\begin{aligned}
\left\|f\left(\frac{p}{2^{n}} x\right)-\frac{p}{2^{n}} f(x)\right\| & \leq \frac{K}{2^{n-1}}\|x\|+\frac{1}{2} \| \\
& f\left(\frac{r}{2^{n-1}} x\right)-\frac{r}{2^{n-1}} f(x) \\
& +f\left(\frac{r+1}{2^{n-1}} x\right)-\frac{r+1}{2^{n-1}} f(x) \| \\
& \leq \frac{K}{2^{n-1}}\|x\|+K \sum_{i=0}^{n-2} \frac{1}{2^{i}}\|x\| \\
& =K \sum_{i=0}^{n-1} \frac{1}{2^{i}}\|x\| .
\end{aligned}
$$

That is, $\|f(\lambda x)-\lambda f(x)\| \leq 2 K\|x\|$ for $0 \leq \lambda \leq 1, x \in X$. 
Theorem 11. Let $f: X \longrightarrow Y$ be an $\epsilon$-bi-Lipschitz map with $f(0)=0$, and with $\epsilon<\frac{1}{3}$. Then $f$ is an almost linear map.

Proof. Let $x, y \in X$. By Proposition 9 and the above Remark

$$
\left\|\frac{f(x)+f(y)}{2}-f\left(\frac{x+y}{2}\right)\right\| \leq \frac{4}{3} K_{1}(3 \epsilon)^{K_{2}}\|x-y\| .
$$

We put $C(\epsilon)=\frac{4}{3} K_{1}(3 \epsilon)^{K_{2}}$. Then

$$
\begin{aligned}
\left\|\frac{1}{2}(f(x)+f(y))-\frac{1}{2} f(x+y)\right\| \leq & \left\|\frac{1}{2}(f(x)+f(y))-f\left(\frac{x+y}{2}\right)\right\| \\
& +\left\|f\left(\frac{x+y}{2}\right)-\frac{1}{2} f(x+y)\right\| \\
\leq & 2 C(\epsilon)(\|x\|+\|y\|) .
\end{aligned}
$$

That is,

$$
\|f(x)+f(y)-f(x+y)\| \leq 4 C(\epsilon)(\|x\|+\|y\|) .
$$

(7) and Lemma 10 imply

$$
\|\lambda f(x)-f(\lambda x)\| \leq 2 C(\epsilon)\|x\|, \text { for } 0 \leq \lambda \leq 1, x \in X .
$$

For each $x \in X$, we define $g_{x}(\lambda)=\|\lambda f(x)-f(\lambda x)\|$ on $R$. Then for $\lambda_{1}, \lambda_{2} \in R$

$$
\begin{aligned}
\left|g_{x}\left(\lambda_{1}\right)-g_{x}\left(\lambda_{2}\right)\right| & =\left|\left\|\lambda_{1} f(x)-f\left(\lambda_{1} x\right)\right\|-\left\|\lambda_{2} f(x)-f\left(\lambda_{2} x\right)\right\|\right| \\
& \leq\left\|\lambda_{1} f(x)-\lambda_{2} f(x)\right\|+\left\|f\left(\lambda_{1} x\right)-f\left(\lambda_{2} x\right)\right\| \\
& \leq 2\left|\lambda_{1}-\lambda_{2}\right|(1+\epsilon)\|x\| .
\end{aligned}
$$

Putting $\lambda_{2}=0$, we obtain

$$
\|\lambda f(x)-f(\lambda x)\| \leq 2|\lambda|(1+\epsilon)\|x\| .
$$

We define $g_{1}(\lambda)=2 \lambda(1+\epsilon)\|x\|$ and $g_{2}(\lambda)=2 C(\epsilon)\|x\|$, for $\lambda \geq 0$. Then $g_{1}$ and $g_{2}$ have the common value at $\lambda=\frac{C(\epsilon)}{1+\epsilon}$. By simple calculation for all $0<\alpha<1$,

$$
\min \left\{g_{1}(\lambda), g_{2}(\lambda)\right\} \leq \frac{8}{3} C(\epsilon)^{1-\alpha} \lambda^{\alpha}\|x\| .
$$

Then (9) and (10) imply

$$
\|\lambda f(x)-f(\lambda x)\| \leq \frac{8}{3} C(\epsilon)^{1-\alpha} \lambda^{\alpha}\|x\|, \text { for } 0 \leq \lambda \leq 1 .
$$

We replace $x$ by $\frac{1}{\lambda} x$ and multiply $\frac{1}{\lambda}(0<\lambda \leq 1)$ in the above formula and we get

$$
\left\|\frac{1}{\lambda} f(x)-f\left(\frac{1}{\lambda} x\right)\right\| \leq \frac{8}{3} C(\epsilon)^{1-\alpha}\left(\frac{1}{\lambda}\right)^{2-\alpha}\|x\| .
$$


Thus

$$
\|\lambda f(x)-f(\lambda x)\| \leq \frac{8}{3} C(\epsilon)^{1-\alpha}\left(\lambda^{\alpha}+\lambda^{2-\alpha}\right)\|x\| \quad \text { for } \lambda \geq 0
$$

Using (8),

$$
\|f(x)+f(-x)\| \leq 4 C(\epsilon)\|x\| .
$$

For $\lambda<0$, put $-\lambda=\beta$. Then

$$
\begin{aligned}
\|\lambda f(x)-f(\lambda x)\| & =\|f(-\beta x)+\beta f(x)\| \\
& \leq 4 C(\epsilon) \beta\|x\|+\frac{8}{3} C(\epsilon)^{1-\alpha}\left(\beta^{\alpha}+\beta^{2-\alpha}\right)\|x\| \\
& \leq \frac{40}{3}\left(C(\epsilon)+C(\epsilon)^{1-\alpha}\right)\left(|\lambda|^{\alpha}+|\lambda|^{2-\alpha}\right)\|x\| .
\end{aligned}
$$

That is,

$$
\|f(\lambda x)-\lambda f(x)\| \leq D(\epsilon, \alpha)\left(|\lambda|^{\alpha}+|\lambda|^{2-\alpha}\right)\|x\|
$$

where $D(\epsilon, \alpha)=\frac{40}{3}\left(C(\epsilon)+C(\epsilon)^{1-\alpha}\right), 0<\alpha<1$. This completes the proof of the theorem.

Let $f$ be an $\epsilon$-bi-Lipschitz map from a finite dimensional Banach space $X$ onto a finite dimensional real Banach space $Y$ with $f(0)=0$. Then there exists a linear map $I$ near $f$.

Theorem 12. Let $X$ and $Y$ be finite dimensional real Banach spaces. If $f: X \longrightarrow$ $Y$ is a surjective $\epsilon$-bi-Lipschitz map with $f(0)=0$ and $\epsilon<\frac{1}{3}$, then there exists a linear isomorphism $I: X \longrightarrow Y$ such that

$$
\|I(x)-f(x)\| \leq E(\epsilon, \alpha)\left(\|x\|^{\alpha}+\|x\|^{2-\alpha}\right)
$$

where $E(\epsilon, \alpha) \rightarrow 0$ as $\epsilon \rightarrow 0$ and $0<\alpha<1$.

Proof. Since $f$ is a homeomorphism from $X$ onto $Y$, there exists a basis

$$
\left\{e_{1}, e_{2}, \ldots, e_{n}\right\}
$$

for $X$ such that $\left\{f\left(e_{1}\right), f\left(e_{2}\right), \ldots, f\left(e_{n}\right)\right\}$ are linearly independent in $Y$ and $\left\|e_{i}\right\|=$ $1, i=1,2, \ldots, n$. We define a surjective linear map $I: X \rightarrow Y$ by

$$
I\left(\sum_{i=1}^{n} \alpha_{i} e_{i}\right)=\sum_{i=1}^{n} \alpha_{i} f\left(e_{i}\right), \quad \alpha_{i} \in R, i=1,2, \ldots, n
$$


If $x \in X$, there exist $\alpha_{1}, \alpha_{2}, \ldots, \alpha_{n}$ in $R$ such that $x=\sum_{i=1}^{n} \alpha_{i} e_{i}$. By Theorem 11,

$$
\begin{aligned}
\|f(x)-I(x)\| & =\left\|f\left(\sum_{i=1}^{n} \alpha_{i} e_{i}\right)-\sum_{i=1}^{n} \alpha_{i} f\left(e_{i}\right)\right\| \\
& \leq\left\|f\left(\sum_{i=1}^{n} \alpha_{i} e_{i}\right)-f\left(\sum_{i=1}^{n-1} \alpha_{i} e_{i}\right)-f\left(\alpha_{n} e_{n}\right)\right\| \\
& +\left\|f\left(\sum_{i=1}^{n-1} \alpha_{i} e_{i}\right)-f\left(\sum_{i=1}^{n-2} \alpha_{i} e_{i}\right)-f\left(\alpha_{n-1} e_{n-1}\right)\right\| \\
& \quad \cdots \\
& +\left\|f\left(\alpha_{1} e_{1}+\alpha_{2} e_{2}\right)-f\left(\alpha_{1} e_{1}\right)-f\left(\alpha_{2} e_{2}\right)\right\| \\
& +\left\|f\left(\alpha_{1} e_{1}\right)-\alpha_{1} f\left(e_{1}\right)\right\|+\left\|f\left(\alpha_{2} e_{2}\right)-\alpha_{2} f\left(e_{2}\right)\right\|+\cdots \\
& +\left\|f\left(\alpha_{n} e_{n}\right)-\alpha_{n} f\left(e_{n}\right)\right\| \\
& \leq(n-1) D(\epsilon, \alpha)\left(\sum_{i=1}^{n}\left|\alpha_{i}\right|\right) \\
& +D(\epsilon, \alpha)\left(\sum_{i=1}^{n}\left(\left|\alpha_{i}\right|^{\alpha}+\left|\alpha_{i}\right|^{2-\alpha}\right)\right) \\
& \leq n^{2} D(\epsilon, \alpha) K\left(\|x\|^{\alpha}+\|x\|^{2-\alpha}\right), \text { for some } K>0 .
\end{aligned}
$$

Put $E(\epsilon, \alpha)=n^{2} D(\epsilon, \alpha) K$. This completes the proof of the theorem.

\section{REFERENCES}

1. J. Gevirtz, Injectivity in Banach spaces and the Mazur-Ulam Theorem on isometries, Trans. Amer. Math. Soc. 274 (1982), 307-318. MR 84h:46024

2. K. Jarosz, Ultraproducts and small bound perturbations, Pacific J. Math. 148 (1991), 81-88. MR 91m:46022

3. F. John, On quasi-isometric mappings, I, Comm. Pure Appl. Math. 21 (1968), 77-110. MR 36:5716

4. S. Mazur and S. Ulam, Sur les transformations isométriques d'espaces vectoriels normés, C.R. Acad. Sci. Paris Sér. 194 (1932), 946-948.

Department of Mathematics, Chungnam National University, Taejon 305-764, Korea E-mail address: kwjun@math.chungnam.ac.kr

Department of Mathematics Education, Konguu National University, Konguu 314-701, Korea 